\title{
Trivium
}

Revue franco-allemande de sciences humaines et sociales - Deutsch-französische Zeitschrift für Geistesund Sozialwissenschaften

$31 \mid 2020$

La culture politique de la République romaine

\section{« Le portrait de son père ». Continuités familiales dans l'historiographie de la République romaine}

\section{Uwe Walter}

Traducteur : Anne-Laure Vignaux

\section{(2) OpenEdition}

Journals

Édition électronique

URL : https://journals.openedition.org/trivium/7133

DOI : $10.4000 /$ trivium.7133

ISBN : 1963-1820

ISSN : 1963-1820

\section{Éditeur}

Les éditions de la Maison des sciences de l'Homme

\section{Référence électronique}

Uwe Walter, « "Le portrait de son père ». Continuités familiales dans l'historiographie de la République romaine », Trivium [En ligne], 31 | 2020, mis en ligne le 25 juin 2020, consulté le 24 août 2021. URL : http://journals.openedition.org/trivium/7133 ; DOI : https://doi.org/10.4000/trivium.7133

Ce document a été généré automatiquement le 24 août 2021.

\section{(c) $(1)$}

Les contenus des la revue Trivium sont mis à disposition selon les termes de la Licence Creative Commons Attribution - Pas d'Utilisation Commerciale - Pas de Modification 4.0 International. 


\title{
« Le portrait de son père ». Continuités familiales dans
}

\section{l'historiographie de la République romaine}

\author{
Uwe Walter \\ Traduction : Anne-Laure Vignaux
}

\section{NOTE DE L'ÉDITEUR}

\section{Édition originale | Originalausgabe}

" "Ein Ebenbild des Vaters". Familiale Wiederholungen in der historiographischen Traditionsbildung der römischen Republik », Hermes, 132, 2004, p. 406-425.

Nous remercions M. Uwe Walter de nous avoir accordé l'autorisation de traduire ce texte pour le présent numéro.

Wir danken Herrn Uwe Walter ür die freundliche Genehmigung, diesen Artikel in französischer Übersetzung zu publizieren.

1 Dans la République romaine, la nobilitas se formait et se reproduisait dans un champ de compétition politique extrêmement complexe, où, à côté de l'origine - la "noblesse " au sens strict - et des actes déjà accomplis, le fait de pouvoir compter à propos d'un candidat à l'élection sur la ligne directrice qu'il allait donner à ses actions futures représentait une importante condition de succès ${ }^{1}$. À cause du peu d'importance que l'on accordait généralement aux programmes concrets, la confiance jouait un rôle essentiel dans les choix électoraux. Comme le fait d'avoir accédé aux degrés inférieurs du cursus honorum n'offrait guère de garanties concernant les capacités d'un candidat dans une fonction de direction, l'élection à cette dernière représentait toujours, aux yeux de l'électeur, un pari sur l'avenir. On pouvait toutefois être certain que le 
détenteur d'une magistrature "fonctionnerait » de façon satisfaisante s'il était garanti qu'il avait acquis le savoir-faire et l'éthique nécessaires par son éducation familiale.

2 À Rome, la socialisation des futurs dirigeants - le plus souvent assurée dans nos systèmes politiques modernes par les partis ou les appareils bureaucratiques - se confondait en bonne partie avec l'éducation des fils par le père dans une maison aristocratique, éducation qui constituait, pour cette raison, un élément central de la culture politique ${ }^{2}$. Son influence était encore renforcée par la patria potestas, laquelle a, en tant qu'instrument de discipline, longtemps rendu superflu et, au bout du compte, retardé le développement d'institutions et de processus "étatiques » à Rome, en particulier dans le domaine judiciaire ${ }^{3}$.

3 La tradition historiographique des débuts de la République ( $\mathrm{V}^{\mathrm{e}}$ et $\mathrm{IV}^{\mathrm{e}}$ siècles) offre un riche réservoir de récits paradigmatiques qui nous éclairent sur la représentation qu'avaient les Romains d'eux-mêmes du point de vue culturel et sociopolitique et la gamme de leurs comportements attendus. Tite-Live les a finalement intégrés en tant que collection d'exemples en leur donnant une force canonique dans son tableau général de l'histoire romaine ${ }^{4}$. Deux d'entre eux font apparaître les possibilités extrêmes du processus de socialisation et du rapport père-fils, évoqués plus haut.

L'épisode de l'élection de P. Licinius Calvus (Esquilinus) comme tribun consulaire en 396 est pétri d'invraisemblances et de contradictions évidentes ${ }^{5}$. Toutefois, Tite-Live l'a, semble-t-il, jugé suffisamment important pour s'accommoder de ces incohérences. Plébéien modéré, Calvus aurait été élu en même temps que d'autres candidats expérimentés alors qu'il n'avait même pas candidaté à la fonction. Il aurait, dès le moment du vote, pris la parole et indiqué qu'en raison de son grand âge et de ses forces déclinantes, il n'était plus à même d'accomplir cette fonction. Mais, aurait-il ensuite ajouté en prenant son fils par la main :

«[...] je vous présente ce jeune homme [...] le portrait, une image fidèle de celui qui, le premier d'entre les plébéiens, obtint de vous le titre de tribun militaire. Ce fils, que j'ai élevé dans mes principes, je le donne et le consacre comme mon remplaçant à la république ; et je vous prie, citoyens, de reporter sur lui cet honneur que vous m'avez déféré sans aucune demande de ma part, et que vous ne refuserez pas à ses sollicitations appuyées de mes prières ${ }^{6}$.»

Le désir du père fut réalisé et $P$. Licinius fut élu - inutile de préciser que le collège, ainsi enrichi du clone de Licinius, s'acquitta brillamment de ses tâches. Un fait important pour la réussite de cette transmission apparaît dans le récit de Tite-Live, à savoir que, physiquement aussi, le fils était la copie conforme de son père (effigies atque imago). Dans une culture où la perception d'ensemble était fortement déterminée par l'élément eidétique, la ressemblance phénotypique pouvait de toute évidence être considérée comme une garantie de la réussite de l'imitation ${ }^{7}$. Cet aspect doit également avoir joué un rôle dans un phénomène abondamment étudié récemment, celui du cortège funèbre (pompa funebris) d'un noble de rang curule qui constituait la mise en scène la plus importante et la plus significative de la continuité et des exploits familiaux. Dans une description bien connue, Polybe insiste expressément sur la «très grande

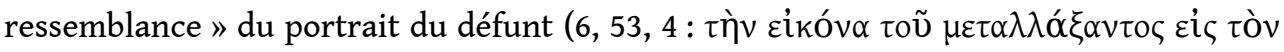

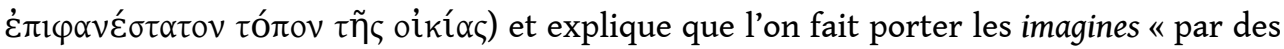
hommes ayant une stature et une corpulence comparables à celles des disparus qu'ils représentent " $(6,53,6)$. Face à la ressemblance physique, trop fréquente pour être fortuite, des fils avec leur père ou leur grand-père, on peut supposer que toutes les 
funérailles de ce type enracinaient dans l'esprit des Romains qui y assistaient les principes évoqués par l'histoire de Licinius.

5 L'autre récit paradigmatique est trop connu pour qu'il soit nécessaire de s'y référer ici en détail : après que les deux fils de L. Brutus et d'autres nobles eurent comploté avec d'autres nobles la restauration du pouvoir des Tarquins - de manière révélatrice, tous de jeunes hommes ${ }^{8}$ - et furent arrêtés, les deux fils de L. Brutus furent jugés et condamnés à mort pour haute trahison; leur père fut, en qualité de consul, chargé de veiller à l'exécution de la sentence ${ }^{9}$. Ce rejet de la politique du père, le plus radical qui pût être, entraînait la peine maximale infligée par celui-ci ${ }^{10}$.

6 Les deux histoires témoignent d'un idéal d'imitation et de conformité familiale qui semblait largement accepté ${ }^{11}$. Dans la res publica, la capacité d'exercice d'une autorité dépendait dans une large mesure de l'accomplissement du rôle paternel, qui incluait l'obligation de transmettre personnellement à ses fils non seulement sa propre vertu, mais aussi l'habitus et les codes du comportement que l'on attendait d'eux. Quand cela réussissait, c'était une source d'honneur suprême, mais en cas d'échec, cela entraînait le désaveu total de la paternité naturelle. La permanence et donc, l'efficacité, de l'influence n'avaient pourtant rien d'évident dans la mesure où de nombreux facteurs contingents et structurels, comme le décès prématuré du père, une séparation ou un remariage et les restructurations familiales qui en résultaient pouvaient se mettre en travers ${ }^{12}$.

7 Dans la tradition historiographique, la norme mimétique esquissée à l'aide de ces exemples se traduisait notamment par l'élaboration, pour les familles éminentes, d'un profil comportemental - un phénomène apparaissant de la façon la plus flagrante chez les Claudii. Des figures marquantes, restées vivantes dans la tradition, offraient aux historiographes des contours fiables qui leur permettaient de modeler d'après cet exemple des parents, souvent homonymes, ou des ancêtres d'un passé plus lointain, dont on ignorait tout sauf, précisément, leur nom ${ }^{13}$. En rendant manifeste la succession des auteurs d'exploits politiques ou militaires, la spectaculaire procession funèbre aristocratique renforçait la supposition de ressemblance et de continuité.

8 La pratique culturelle et la représentation de l'ordre politique et social se faisaient mutuellement référence. Du point de vue de la sociologie de la domination, ces manifestations, parce qu'elles mettaient en scène la longue continuité générationnelle et les succès militaire des familles au pouvoir, ont sans aucun doute stabilisé le régime aristocratique et instillé auprès du populus Romanus cette confiance qui explique en bonne partie l'obéissance étonnamment durable à l'égard de la nobilitas ${ }^{14}$.

9 Je me propose de commenter les facteurs associés à l'idéal sociopolitique tel qu'il est esquissé, de la ressemblance et de l'imitation, ainsi que de l'addition et de la répétition comme modèles d'engendrement (generative patterns) lors de la formation de la tradition historiographique. On peut distinguer cinq variantes de ces modèles. Dans les pages qui suivent, elles seront présentées à travers des exemples connus dans le but de faire ressortir le rapport étroit entre tradition, conformité des rôles et prise de décision.

10 1. Le facteur du pouvoir représente sans aucun doute un paradigme de référence pour la représentation et la périodisation de l'histoire des débuts de la république. La mise en sécurité du nouvel ordre face aux ambitions rivales des Tarquins et des Porsenna, l'emploi rapide et déterminé de la force par les patriciens après que cette offensive eut 
été repoussée et, enfin, la formation d'un contre-pouvoir plébéien sont considérés dans ce cadre comme les étapes importantes. Le pouvoir des patriciens se manifesta bien entendu dans les récits par, d'un côté, le monopole qu'ils exerçaient naturellement sur les magistratures et, de l'autre, par la fermeture de leur organisation en groupes familiaux. Il faut mentionner ici la narration de référence que constituait l'arrivée à Rome d'Attus Clausus avec 5000 clients en 504 av. J.-C. ${ }^{15}$. Attus (= Appius) passe pour le fondateur de la gens romaine à laquelle on attribuerait plus tard le profil familial le plus singulier. Le fait que la gens patricienne des Claudii fut peut-être sinon la plus célèbre, du moins la plus constante au plus haut niveau de la Rome républicaine et que en même temps elle ait été caractérisée par des stéréotypes - aucune autre famille romaine n'a été identifiée avec autant de cohérence et de clarté sous la république romaine - ne forme que les deux faces d'une même réalitée ${ }^{16}$.

11 L'existence de plusieurs fils similaires, égaux en vertu et en noblesse d'esprit confère également de la puissance à un aristocrate ${ }^{17}$. À cette condition, l'addition et la répétition déterminent l'étape suivante de l'« histoire du pouvoir " propre aux débuts de la république : une fois le terrain préparé pour deux conflits durs et durables - d'une part, la première sécession de la plèbe et l'exécution de Sp. Cassius, de l'autre, la reprise des hostilités avec Véies -, les Fabii s'imposent à la tête des opérations dans les deux cas. De 485 à 479, un Fabius est à chaque fois investi du consulat - trois frères, soudés par la pietas, tous faits d'acier trempé, se partagent sept mandats ${ }^{18}$. Nous atteignons le niveau le plus élevé de l'idéalisation du pouvoir familial, également exprimée dans la désignation collective de Fabium nomen dans la légende de l'expédition des 306 Fabii, qui se chargèrent avec leurs clients de combattre les Véiens et tombèrent dans une embuscade sur les rives du Cremère ${ }^{19}$. La refonte socioculturelle du motif est ici caractéristique : si, dans les Thermopyles, 300 citoyens hoplites de Sparte avaient été placés sous les ordres de l'un des deux détenteurs de la fonction suprême, on observe dans l'histoire de Fabii la prédominance ou du moins la plausibilité d'une norme gentilice radicalisée. Ainsi, pendant le siège de Rome par les Gaulois, un Fabius aurait quitté la sécurité du Capitole et se serait exposé à l'ennemi dans le seul but d'accomplir un acte rituel institué par sa famille ${ }^{20}$. Les exemples fabiens de symbiose politique entre père et fils remontent à des temps mieux connus : elle est frappante entre Q. Fabius Maximus Cunctator et son fils dans la guerre d'Hannibal ${ }^{21}$. L'énergie avec laquelle Fabius Maximus Rullianus, arrivé à un âgé avancé, proposa son fils Gurges comme légat en 292, trente ans après son premier consulat, a souvent été mise en avant dans la tradition. Celui qui avait associé son fils à son triomphe quand celui-ci n'était qu'un gamin marchait à présent derrière lui dans ce triomphe ${ }^{22}$. Dans l'historiographie, cette présomption de ressemblance pouvait même être exploitée, à des fins "méthodologiques", pour résoudre une question litigieuse. Ainsi, pour l'année 302, deux noms se présentaient à Tite-Live dans les sources en lien avec la fonction de magister equitum : M. Aemilius Paullus et Fabius Maximus Rullianus, un homme déjà très célèbre, que nous venons de nommer. Mais comme le chef de la cavalerie était tombé dans une embuscade pendant qu'il était allé au fourrage et avait perdu de nombreux soldats, Tite-Live crut pouvoir choisir : une telle erreur de jugement était impensable pour un Fabius, donc c'était l'autre qui était le malchanceux ${ }^{23}$.

12 Le «modèle fabien » ne resta d'ailleurs pas limité à l'aristocratie patricienne. Dans la tradition populaire-plébéienne, il y avait également, à côté d'individus qui se distinguaient, des lignées composées d'hommes très semblables au cours d'une période donnée. S'il était évident que le Licinius Calvus, déjà cité, devait agir en politique main 
dans la main avec son fils, des constructions plus marquantes ne manquent pas non plus. Ainsi, au Ve siècle, certains tribuns de la plèbe de la gens Icilia, qui s'est éteinte plus tard, ont joué un certain rôle et dans l'œuvre de Tite-Live ont été ramenés au type de tribuns rebelles et amoureux de la liberté24.

Un certain nombre d'actions et d'initiatives importantes intervenues sur trois générations sont réparties de manière assez arbitraire entre deux Lucii et un Spurius. En 409, il y a même, semble-t-il, trois tribuns de la plèbe appartenant à la gens Icilia ${ }^{25}$ il s'agit, comme dans le cas des Fabii, d'un reflet historiographique du fait que plusieurs magistrats venant d'une même famille en l'espace d'une génération augmentaient considérablement ses chances politiques, comme $\mathrm{H}$. Beck vient de le montrer grâce à l'exemple des Atilii durant la Première Guerre punique ${ }^{26}$. La seule chose qui importe ici, c'est un constat général valable également pour les cas suivants: dans l'optique de la tradition, l'aristocratie, bien qu'en réalité elle fût structurée et recrutée de manière tout autre dans la période des débuts, «fonctionnait », d'après les sources, sur le même modèle que l'aristocratie des $\mathrm{III}^{\mathrm{e}}$ et $\mathrm{II}^{\mathrm{e}}$ siècles, autrement dit de l'époque où l'historiographie romaine, puis latine a fait son apparition, où, comme dans le cas des Atilii, des sortes de «niches familiales » dans les listes de consuls restaient un souvenir vivant et où, au même moment, les discours et éloges politiques étaient documentés et conservés. En ce qui concerne les sources, une conclusion importante se dégage: comme nous possédons pour la plus grande partie du III siècle aussi peu de sources latines détaillées que pour l'époque des Gracques et de Sylla, la première décade de Tite-Live offre, pour la sémantique et la rhétorique politiques - en particulier familiales - de cette époque, un témoignage non négligeable.

14 2. Le modèle suivant était étroitement lié à une pratique courante de la culture historique romaine: les aristocrates romains se sentaient particulièrement responsables de la présence durable de l'héritage de leurs ancêtres. Ils rénovaient les temples et les autres constructions ou monuments que ces derniers avaient consacrés ou bâtis. Dans le cas des bâtiments utilitaires, comme les basiliques, le lien nominal était particulièrement significatif. Comme il en allait de même pour les lois, les récits des historiens concernant l'engagement de certains pour l'héritage politique d'un ancêtre n'ont rien de surprenant. Le tribun de la plèbe $\mathrm{Cn}$. Trebonius se serait ainsi battu en 401 pour qu'un plébiscite trébonien plus ancien, relatif à l'élection des tribuns de la plèbe, fût également respecté. Selon Tite-Live, il devait «à son nom et à sa famille ${ }^{27}$ " la défense de cette loi. En 326, les consuls C. Poetelius Libo Visolus et L. Papirius Cursor firent passer une loi qui interdisait de retenir les débiteurs dans les chaînes en cas d'emprisonnement pour dettes, comme le stipulait la loi des Douze tables. En 313, le fils homonyme de Poetelius acheva en qualité de dictateur le travail de réforme de son père, qui avait fait partie grâce à son triple consulat $(360,346,326) d u$ premier rang de l'élite plébéienne, en abolissant entièrement par une loi l'asservissement pour dettes ${ }^{28}$. L'évidence que l'on pouvait attendre d'un tel comportement reposait sur un modèle d'engendrement : à chaque fois que la tradition offrait un nom, il était possible, dans les conditions évoquées, d'extrapoler de façon plausible un acte politique.

15 L'exemple le plus connu de répétition d'un acte politique nous est fourni par la tradition relative à la législation sur la provocatio ad populum, appel adressé par les citoyens romains à l'ensemble du peuple à contester la juridiction capitale d'un magistrat. Le droit de provocation, désigné à plusieurs reprises comme le palladium de 
la liberté, est indissociablement lié aux noms de Valerius et d'Horatius. En 509, le consul P. Valerius Publicola voulut, selon un récit que personne ne remettait en question à Rome, faire adopter une première loi réduisant le pouvoir illimité, quasi monarchique, du consul ${ }^{29}$. Cette loi fut affinée et élargie par les consuls L. Valerius Potitus et M. Horatius Barbatus en 449 , après la chute du second décemvirat tyrannique ${ }^{30}$. Tite-Live note en 300 une proposition de loi de M. Valerius relative à la provocation et observe qu'il s'agit de la "troisième proposée depuis l'expulsion des rois, et toujours par la même famille» (Liv. 10, 9, 3) $)^{31}$. Les prémisses, les faits et les conclusions sont amalgamés en un résultat plausible. La loi - par ailleurs mal documentée - de 300 avait été imposée par un membre de la famille des Valerii et le cognomen du légendaire P. Valerius Publicola («soucieux du peuple ») conduisait à la conclusion d'une sympathie générale des Valerii patriciens pour le peuple ${ }^{32}$, ce qui situait ses membres aux antipodes des Claudii. Dans l'optique de l'historiographie, les Valerii étaient associés à certains thèmes et à un certain style politique. Le fait qu'avant et après la fondation de l'historiographie romaine par Fabius Pictor, une part essentielle de la mémoire historique ait été conservée et déterminée par les différentes gentes encourage également à relier en pensée les sommets des ancêtres célèbres ${ }^{33} \mathrm{en}$ intégrant au passage quelques collines plus modestes.

16 3. Dans le cas des lois de provocation valério-horaciennes, l'analyse concrète montre qu'une telle mesure n'était probablement imaginable qu'en l'an 300, au moment de l'établissement d'une forme de justice capitale acceptée de tous, neutre en termes de classes sociales, qui supplantait les deux modèles concurrents de la "période du conflit» antérieure - c'est-à-dire la coercition des magistrats et les tribunaux d'exception de la plèbe. Après son acceptation comme une loi fondamentale de la res publica, il paraissait naturel de relier également la provocation à la fondation et à la refondation de l'État - d'autant qu'avec les Valerii et les Horatii, des acteurs tout trouvés étaient à disposition pour les deux périodes. De tels repères structuraient et périodisaient le champ embrouillé des événements.

17 Cependant, on a voulu voir un nombre bien plus élevé de ces prétendus doublons dans les innombrables guerres et batailles dont la première et la troisième décades de TiteLive, en particulier, regorgent ${ }^{34}$. Nous ne pensons pas ici aux cas évidents où la même affaire est racontée deux fois sur un court laps de temps ${ }^{35}$, mais à ces événements qui comme les lois de provocation - ont été considérés comme des dédoublements inventés d'un événement généralement postérieur impliquant des acteurs d'une même famille et ont, pour cette raison, été écartés. Citons au hasard la conquête, associée à l'an 317, de la ville de Nerulum par C. Iunius Brutus Bubulcus comme projection rétrospective anhistorique du triomphe, indubitable celui-là, célébré par le fils homonyme de Iunius sur les Lucaniens en $277^{36}$. L'usage de cette argumentation comme passe-partout n'est pourtant pas sans poser certains problèmes, du moins pour le IV ${ }^{\mathrm{e}}$ siècle (moins pour le $\mathrm{V}^{\mathrm{e}}$ ). Nous devons garder à l'esprit que le nombre de familles consulaires n'était pas très élevé et qu'il pouvait sembler raisonnable d'envoyer le fils ou le neveu d'un détenteur d'imperium qui avait fait ses preuves sur le même théâtre d'opérations - que ce soit en raison de sa connaissance de la situation ou de la confiance en une perpétuation des compétences familiales. Pour affiner encore l'argument: une chasse mécanique aux doublons devrait également amener à considérer comme invraisemblable que deux Cornelii Scipiones aient vaincu Carthage en 202 et 146 et qu'en plus, quatre hommes de ce nom, appartenant à trois générations, se soient battus en Espagne ${ }^{37}$. Cette mise en garde vaut aussi, mutatis mutandis, pour le domaine législatif, même si les trois leges 
Porciae mentionnées par Cicéron ne sont dans l'ensemble que faiblement documentées ${ }^{38}$. Comment envisagerait-on les informations sur la législation de Tiberius et Caius Gracchus si elles étaient évoquées dans le quatrième livre de Tite-Live ?

Le problème se pose de manière plus aiguë encore quand nous sommes confrontés à la répétition d'un acte remarquable. Nous pouvons citer ici les célèbres sacrifices rituels (devotiones) des trois Publii Decii Mures. Confrontés tous trois à une situation critique pour l'armée romaine, le père, le fils et le petit-fils auraient à chaque fois répété la même formule de dévotion auprès d'un prêtre et cherché la mort dans le combat afin d'entraîner l'ennemi dans sa chute: en 340 lors de la bataille du Veseris, en 295 à Sentinum et en 279 à Ausculum ${ }^{39}$. Même en l'absence de toute certitude, il semble difficile de reléguer ces trois récits dans le domaine des fables. Il est en tout cas assez probable que le père, premier consul de sa famille en 340, ait trouvé la mort sur le champ de bataille; le fait est pratiquement certain pour son fils, l'un des grands généraux de la troisième guerre samnite et consul à quatre reprises. Son contemporain Duris de Samos mentionnait déjà ce sacrifice en même temps que le chiffre considérable et exagéré - de 100000 morts ennemis ${ }^{40}$. Le même auteur semble avoir déjà parlé au pluriel des Decii qui s'étaient sacrifiés ${ }^{41}$. Les Decii Mures conquirent une place stable dans l'histoire populaire romaine au $\mathrm{II}^{\mathrm{e}}$ siècle au plus tard lorsqu'ils furent à la fois célébrés par Ennius dans ses Annales et par Accius dans un drame historique intitulé Decius. Le second fait dire à son Decius, celui de 295 : «Suivant l'exemple de mon père, je vais consacrer ma personne et dévouer ma vie pour la perte des ennemis $^{42}$. » La conscience historique populaire, façonnée par les lectures scolaires et la scène, telle que la représente, par exemple, Cicéron, pouvait naturellement ignorer un problème: la bataille d'Ausculum, livrée contre Pyrrhus en 279, était une défaite romaine. Or il ne pouvait pas y avoir de dévotion infructueuse. Par ailleurs, certains indices suggèrent que le troisième Decius ne trouva pas du tout la mort à cette occasion, ou, qu'en tout cas, il ne se produisit pas de dévotion réussie à Ausculum ${ }^{43}$. Pour sauver la série, les historiographes ont eu recours à des artifices aventureux, affirmant que Pyrrhus avait ordonné de ne tuer en aucun cas un Romain dans une certaine tenue qui méprisait la mort, mais seulement de le faire prisonnier. Mieux encore, une correspondance aurait été échangée au sujet de la dévotion envisagée et Decius aurait finalement renoncé à son projet. Mais pour tous les non-historiens, ce n'étaient là que des détails sans importance ; les signaux évidents de la série avaient davantage de poids : père, fils, petit-fils tous trois consuls et portant le même prénom de Publius, tous les trois - et ils étaient les seuls chez les Decii ! - avec le cognomen de Mus $^{44}$ et participant à une grande bataille contre un ennemi au départ supérieur. Pour l'historien, il était également tout à fait évident qu'une telle dévotion avait des conséquences obligatoires sur les actes de la descendance. Elle représentait surtout un atout politique à exploiter : lorsqu'en l'an 300, le débat politique intérieur à Rome porta sur le fait de briser le monopole patricien de la prêtrise, le plébéien Decius Mus - dont l'adversaire était évidemment un Claudius - « rappela, dit-on, l'image de son père tel que beaucoup de ceux qui étaient à l'assemblée l'avaient vu : la toge ceinte selon le rite gabien, debout sur un javelot, dans l'attitude où il se dévoua pour le peuple et les légions romaines » [trad. française : Eugène Lasserre, Garnier, 1937]. Et un homme qui était apparu si pur et si agréable aux dieux aurait été, selon le droit en vigueur, inapte à une fonction de prêtre ${ }^{45}$ ? Comme on le voit, un acte et une obligation qui était d'une certaine façon limitée tout à fait hermétiquement aux Decii Mures se laissa en même temps convertir en capital politique. Ce qui apparaissait déjà dans les exemples de 
Licinius Calvus, des Fabii et des Icilii trouve ici une confirmation: on ne peut pas commettre le contresens d'interpréter la communication de l'aristocratie avec le peuple dans des catégories extrêmes comme des groupements fixes et des blocs de clientèles, ou comme un populisme démocratique, mais on peut identifier des structures stables, qui rendaient les acteurs prévisibles et leur comportement saisissable et attractif. Et l'on peut difficilement omettre le fait que des engagements ou, mieux, des contraintes y furent liées.

19 4. La question de savoir si un de ces sacrifices personnels, voire plusieurs ont réellement eu lieu et $\mathrm{si}$, donc, le troisième Decius a réellement entrepris cette vaine tentative de s'inscrire dans une série déjà existante est insoluble. D'un point de vue structurel, il s'agit en tout cas d'un schéma de répétition d'une extrême simplicité. L'histoire est nettement plus complexe quand on s'intéresse à certains Manlii Torquati des $\mathrm{IV}^{\mathrm{e}}$ et $\mathrm{II}^{\mathrm{e}}$ siècles. On peut décrire ce schéma d'imitation comme un modèle définissant une personnalité avec les actes correspondants. Les acteurs sont au nombre de cinq :

L. Manlius Imperiosus, dictateur en 363, fut apparemment accusé par un tribun de la plèbe parce qu'il avait fait honneur à son cognomen et non seulement, il avait levé des soldats avec une grande dureté, mais qu'en outre, il avait banni son fils et l'avait condamné à des travaux serviles à la campagne sans raison valable ${ }^{46}$.

Ce fils, Titus, quitta précipitamment sa charrue pour se rendre à Rome et, brandissant un couteau, somma chez lui le tribun de la plèbe d'abandonner son accusation. Plus tard, en tant que tribun militaire, il affronta avec la permission expresse du consul un guerrier gaulois en combat singulier et le tua. Le torque ensanglanté, qu'il ramena en guise de trophée, lui procura le surnom honorifique de Torquatus ${ }^{47}$.

Lorsque ce T. Manlius Torquatus fut consul pour la troisième fois en 340 - par ailleurs en même temps que le premier Decius Mus -, il fit semble-t-il exécuter son fils qui portait le même nom après un combat victorieux parce que celui-ci avait provoqué un commandant de la cavalerie latin en duel ${ }^{48}$. Il avait certes réussi à tuer son adversaire, mais il avait agi contre les ordres, qui étaient de ne pas quitter les rangs. À l'imitation de son père, le consul sanctionna ainsi la tentative inopportune de son fils d'imiter son propre père alors que son collègue Decius Mus donnait au même moment à son fils un exemplum positif, quoique se soldant également par la mort ${ }^{49}$.

En 140, T. Manlius Torquatus bannit son ancien fils, entre-temps adopté par une autre famille, parce que celui-ci avait été accusé de détournement d'argent en tant que préteur en Macédoine et ne s'était donc pas montré digne de ses ancêtres dans l'exercice de sa fonction ${ }^{50}$. Le père biologique avait auparavant prié le Sénat de ne rien entreprendre avant qu'il ne fût parvenu à une décision dans une procédure de jugement domestique (judicium domesticum). Étant donné que depuis son passage dans la famille des Iunii Silani, son fils n'était plus soumis à la patria potestas de Torquatus, il faut supposer que le sénat l'avait remis à son père pour la procédure. Le pouvoir du père n'était pas pour autant rétabli ; à défaut de pouvoir prononcer un jugement légal, Torquatus ne put entreprendre qu'une cognitio. Arrivé à la conclusion que son fils était coupable, il le déclara à la fois indigne de l'État et de la maison des Torquati et le chassa de chez lui.

24 Le fils ne vit d'autre issue que le suicide, devenant ainsi, consciemment, le fantôme des fils Manlius. De surcroît, il choisit la pendaison, une forme de suicide réservée aux femmes. Silanus s'humiliait ainsi de manière significative; il était manifestement 
convaincu qu'il ne pouvait exprimer autrement sa honte. Son père biologique n'assista pas aux funérailles, mais donna audience à ceux qui voulaient le consulter en droit dans l'atrium de sa maison, en présence des images de ces Manlii qui portaient le surnom d'Imperiosus ( «impérieux »). Lors du procès domestique qui avait eu lieu dans l'atrium, les imagines maiorum avaient représenté le consilium de la famille, ce qui est habituel en pareille circonstance.

Nous avons ici une correspondance claire entre une sévérité paternelle qui ne doit pas se justifier et le respect du fils vis-à-vis de son père, auquel il doit aide et obéissance et dont il accepte l'autorité sans contestation ${ }^{51}$. Même le fait de menacer un tribun de la plèbe, en soi un crime grave, fit honneur au futur Torquatus dans la mesure où « toute la rigueur paternelle n'avait pu rebuter la pieuse tendresse de son âme» (Liv. 7, 5, 7). En tant que fils, il accepta et défendit le comportement que son rôle imposait à son père, qu'il devait reproduire plus tard de manière plus brutale encore. Avoir du sang des Manlii dans les veines signifiait en tant que père être dur, voire impitoyable et faire obéir son fils en toute circonstance, et, en tant que fils, accepter toutes les conséquences de cette sévérités ${ }^{2}$. Dans ce cycle fermé de légendes du IV siècle, les comportements définis par les rôles et les fonctions se recoupent de manière trop exacte pour que le message ne soit pas évident; les Manlii Torquati comptaient d'ailleurs parmi les exempla fréquemment cités. Maurizio Bettini affirme qu'au fil de trois générations, la répétition des actes fonde le modèle ni plus ni moins mythifié de la relation père-fils. En tant que tel, ce modèle faisait naturellement partie du discours de pouvoir propre à la société patriarcale, lequel impliquait que le droit de mise à mort du pater familias sur ses fils, bien que peu revendiqué dans les faits, n'ait jamais été aboli formellement. Tandis que le Romain "normal» d'une période postérieure pouvait frissonner et reculer face aux Manliana exempla, le modèle de comportement lié de manière emblématique au nom d'une famille déterminée continuait à représenter un impératif pour les membres de la famille en question, d'autant que l'exemple était généralisable, outre la relation père-fils, à la severitas exemplaire. En 216, par exemple, le vétéran sénatorial T. Manlius Torquatus (consul pour la première fois en 235), un homme priscae ac nimis durae, ut plerisque videatur, severitatis (Liv. 22, 60, 5), s'opposa avec véhémence au rachat de Romains fait prisonniers par les Carthaginois. Dans ce cas, comportement réel et formation de la tradition se laissent suivre en parallèle : l'objection en tant que telle est historiquement assez vraisemblable, tandis que TiteLive a construit le discours librement, mais en le fondant sur des arguments attendus; les exempla historiques ont joué dans ce cadre un rôle essentiel ${ }^{53}$. Et même si les pères de famille romains "normaux » ne se comportaient certainement pas comme Manlius Torquatus, le simple fait que quelque chose de tel avait pu se produire était resté si marquant dans les mémoires et était de surcroit accepté suffisait à créer une atmosphère très particulière, dans laquelle se produisait la socialisation de chaque nouvelle génération de futurs dirigeants.

Le nom était en tout cas à même d'exercer un réel pouvoir et la répétition délibérée de ces actes extrêmes, comme la répudiation du fils et son suicide en 140, doit également inciter à se garder, pour les périodes antérieures également, de crier systématiquement et par réflexe au doublon sous le prétexte d'un scepticisme éclairé ${ }^{54}$. Que ce soit pour les Manlii ou pour les Decii Mures, il est préférable de tabler sur un va-et-vient complexe entre norme sociale, gestion réelle des événements et du souvenir historique et, enfin, formation de la tradition. 
5. Le modèle de pensée familiale était si fermement ancré et en même temps si influent que, durant la république tardive, il a pu être utilisé dans un contexte entièrement différent : des aristocrates sans autre capital notable se servaient d'un ancêtre célèbre pour individualiser leur politique et leur habitus avec une certaine extravagance et ainsi faire accepter ou au moins attirer l'attention sur un comportement personnel qui était souvent consciemment non conforme à la règle. Une telle construction d'un profil individuel pouvait parfois avoir des conséquences sur l'existence plus ou moins analogues à celles du cas, déjà cité, du Manlianus tardif, mais il avait aussi, et bien plus que dans ce cas, un caractère instrumental.

Alors que des descendants des familles vénérables se voyaient à l'occasion rappeler le souvenir de leurs ancêtres en tant d'exemples contraignants ${ }^{55}$, et étaient parfois même accusés de ne pas connaître leur propre histoire familiale ou d'être entièrement dégénérés sur le plan politico-moral, d'autres s'employaient à une confirmation très ciblée de la tradition, parfois même à une construction de celle-ci. On peut citer comme exemples pertinents des gens comme Marcus Porcius Cato Uticensis et Marcus Iunius Brutus, à qui une schématisation délibérée à partir de l'attitude d'un ancêtre illustre a offert une marge de manœuvre étonnement large : Caton le Jeune, arrière-petit-fils du célèbre censeur, pouvait ainsi se présenter comme un compagnon rigide et quelquefois totalement hors du temps sans perdre pour autant la reconnaissance et le respect; il jouissait aussi, sans doute dès avant son suicide, d'un prestige politique qui dépassait de loin celui que son rang sénatorial aurait dû lui valoir ${ }^{56}$. Quant à Brutus, il a pu apparaître comme un ennemi déclaré des tyrans dans un milieu de potentats et gagner un prestige et une influence que ses fonctions ne lui conféraient pas non plus ${ }^{57}$. Mais avec la construction résolue d'un profil personnel qui semblait aboutir à l'affirmation d'un programme et d'une idéologie selon un mode qui n'avait rien de nobiliaire, tous deux se sont en revanche privés de possibilités d'action alternatives dans des situations politico-morales exceptionnelles.

On peut voir dans les quatre premiers modèles les variantes d'un unique modèle d'engendrement pour l'organisation narrative des débuts de l'histoire romaine à travers l'historiographie. Il s'agissait du quasi-monopole familial sur certaines fonctions et de l'action concertée à la fois dans la génération présente et dans les suivantes, de la défense active de l'héritage politique et de la mémoire d'un ancêtre, de la répétition d'un acte donné sur plusieurs générations et, enfin, de la construction créatrice d'exemplum d'un modèle de comportement déterminé visant à intérioriser une conception centrale de l'ordre social. Néanmoins, l'exemple des Manlii montre aussi avec quelle facilité la dynamique mythique peut générer une directive, voire une contrainte de comportement. Cet aspect domine complètement dans le modèle que nous venons d'esquisser, qui n'appartient pas par hasard à la fin de la République, autrement dit à l'époque de la désintégration : chez Caton et Brutus, le nom n'avait plus réellement de dimension familiale et aucun code nobiliaire n'a plus non plus été activé ; le lointain ancêtre (qui dans le cas de Brutus en fait n'existait pas) ne servait plus qu'à la dynamique individuelle et finissait de ce fait par avoir la même fonction que la dignitas de César et la prétention de Cicéron à défendre le bien commun seul et à titre personnel. Si l'on voulait décrire une évolution à travers les cinq modèles nommés, celle-ci irait du comportement familial comme impératif légitime de la formation de la tradition à la tradition familiale comme impératif légitimant du comportement. 
modèles décrits ne permettront pas de fabriquer un détecteur de mensonges à l'usage de la critique des sources pour les débuts de la République. La formation de la tradition - rendue plus complexe encore par le système romain des noms - reste, tout au long des étapes menant de la tradition orale et des souvenirs familiaux spécifiques à l'historiographie, une jungle difficile à pénétrer dans la mesure où les pratiques et les normes sociopolitiques concrètes étaient également des mécanismes générateurs d'histoire et inversement. Cependant, les chemins tracés par les Romains à l'ère de la nobilitas ont peut-être gagné en lisibilité ${ }^{58}$. Si je suis en outre parvenu à dégager de la banalité d'un phénomène superstructurel la conscience historique des Romains, dont l'historiographie représente la forme d'expression la plus saisissable, bien que certainement pas l'unique, pour la présenter au contraire, en m'appuyant sur un contexte spécifique de la culture politique romaine, comme une force qui guide les actes et façonne la société, alors ces considérations auront atteint leur objectif ${ }^{59}$.

\section{BIBLIOGRAPHIE}

\section{Abréviations}

FRH : Die Frühen Römischen Historiker. Vol. 1: Von Fabius Pictor bis Cn. Gellius ; Vol. 2 : Von Coelius Antipater bis Pomponius Atticus. Édité, supervisé et commenté par H. Beck et U. Walter, Darmstadt, Wissenschaftliche Buchgesellschaft, 2001 et 2004.

MRR : T. R. S. Broughton, The Magistrates of the Roman Republic, vols. I/II, New York, The American Philological Association, ${ }^{2} 1968$; III, Atlanta, 1986.

\section{Literatur}

Bannon, C. (1997) : The Brothers of Romulus. Fraternal Pietas in Roman Law, Literature, and Society, Princeton, Princeton University Press.

Beck, H. (2005) : Karriere und Hierarchie. Die römische Aristokratie und die Anfänge des cursus honorum in der mittleren Republik, Berlin, Akademie Verlag.

Beloch, K. J. (1926) : Römische Geschichte, Berlin/Leipzig, De Gruyter.

Bettini, M. (1991) : Anthropology and Roman Culture. Kinship, Time, Images of the Soul, Baltimore/ Londres, Johns Hopkins University Press.

Bleicken, J. (1959) : « Ursprung und Bedeutung der Provokation », ZRG, 76, p. 324-377.

Blösel, W. (2003) : « Die memoria der gentes », in : Eigler, U. et al. (éd.) : Formen römischer Geschichtsschreibung von den Anfängen bis Livius, Darmstadt, Wissenschaftliche Buchgesellschaft, p. 53-72.

Bradley, K. (1991) : Discovering the Roman Family : Studies in Roman Social History, Oxford, Oxford University Press. 
Chaplin, J. (2000) : Livy's Exemplary History, Oxford, Oxford University Press.

Clemente, G. (1990) : « La politica romana nell'età dell'imperialismo », in : Schiavone, A. (éd.) : Storia di Roma, II 1, Turin, Einaudi, p. 235-266.

Cornell, T. J. (1995) : The Beginnings of Rome, Londres, Routledge.

Dondin-Payre, M. (1993) : Exercice du pouvoir et continuité gentilice : les Acilii Glabriones du IIIe siècle avant J.-C. au Ve siècle après J. C., Rome, École française de Rome.

Elster, M. (2003) : Die Gesetze der mittleren römischen Republik, Darmstadt, Wissenschaftliche Buchgesellschaft.

Fehrle, R. (1983) : Cato Uticensis, Darmstadt, Wissenschaftliche Buchgesellschaft.

Flach, D. (1994) : Die Gesetze der frühen römischen Republik, Darmstadt, Wissenschaftliche Buchgesellschaft.

Flaig, E. (1995) : « Die Pompa funebris. Adlige Konkurrenz und annalistische Erinnerung in der römischen Republik », in : Oexle, O. G. (éd.) : Memoria als Kultur, Göttingen, Vandenhoeck \& Ruprecht, p. 115-148.

Flaig, E. (2003) : Ritualisierte Politik. Zeichen, Gesten und Herrschaft im Alten Rom, Göttingen, Vandenhoeck \& Ruprecht.

Hölkeskamp, K.-J. (1987) : Die Entstehung der Nobilität, Wiesbaden, Franz Steiner.

Ihne, W.(1893) : Römische Geschichte, vol. 1, 2éd., Leipzig, Engelmann.

Jehne, M.(1995) : « Die Beeinflussung von Entscheidungen durch "Bestechung”: Zur Funktion des Ambitus in der römischen Republik », in : id. (éd.) : Demokratie in Rom? Die Rolle des Volkes in der Politik der römischen Republik, Stuttgart, Franz Steiner, p. 51-76.

Jehne, M. (2002) : « Die Geltung der Provocation und die Konstruktion der römischen Republik als Freiheitsgemeinschaft », in : Melville, G. / Vorländer, H. (éd.) : Geltungsgeschichten. Über die Stabilisierung und Legitimierung institutioneller Ordnungen, Cologne et al., Böhlau, p. 55-74.

Linke, B. (1995) : Von der Verwandtschaft zum Staat, Stuttgart, Franz Steiner.

Lintott, A. W. (1972) : « Provocatio. From the Struggle of the Orders to the Principate ", ANRW, 1.2, p. 226-267.

Martin, J. (2002a) : « Familie, Verwandtschaft und Staat », in : Spielvogel, J. (éd.) : Res publica reperta (FS Bleicken), Stuttgart, Franz Steiner, p. 13-24.

Martin, J. (2002b) : « Formen sozialer Kontrolle im republikanischen Rom », in : Cohen, D. (éd.) : Demokratie, Recht und soziale Kontrolle im klassischen Athen, Munich, Oldenbourg, p. 155-172.

Mommsen, Th. (1864) : « Die patricischen Claudier », Römische Forschungen I, Berlin, p. 285-318.

Mommsen, Th. (1887) : Römisches Staatsrecht, $3^{\mathrm{e}}$ éd., Leipzig, S. Herzel.

Niebuhr, B.G. (1843) : Römische Geschichte, vol. 3, $2^{\mathrm{e}}$ éd., Berlin, Realschulbuchhandlung.

Oakley, S. P. (1997) : A Commentary on Livy, Books VI-X, vol. 1 : Introduction and Book VI, Oxford, Clarendon Press.

Oakley, S. P. (1998) : A Commentary on Livy, Books VI-X, vol. 2 : Books VII-VIII, Oxford, Clarendon Press.

Ogilvie, R. M. (1970) : A Commentary on Livy 1-5, 2e éd., Oxford, Oxford University Press.

Pais, E. (1906) : Ancient Legends of Roman History, Londres, Swann Sonneschein. 
Plescia, J. (1976) : « Patria potestas and the Roman revolution », in : Bertman, S. (éd.) : The Conflict of Generations in Ancient Greece and Rome, Amsterdam, B.R. Grüner, p. 143-169.

Richard, J.-C. (1972) : « Qualis pater, talis filius », Revue de philologie, 46, p. 43-55.

Richard, J.-C. (1989) : « L'affaire du Crémère. Recherches sur l'évolution et le sens de la tradition ", Latomus, 48, p. 312-325.

Richard, J.-C. (1990) : « Historiographie et histoire : L'expédition des Fabii à la Crémère », in : Eder, W. (éd.) : Staat und Staatlichkeit in der frühen römischen Republik, Stuttgart, Franz Steiner, p. 174-199.

Richardson, J. H. / Santangelo, F. (éd.) (2014) : The Roman Historical Tradition. Regal and Republican Rome, Oxford, Oxford University Press (Oxford Readings in Classical Studies).

Scholz, P. (2011) : Den Vätern folgen. Sozialisation und Erziehung der republikanischen Senatsaristokratie, Berlin, Antike Collection.

Schwegler, A. (1884) : Römische Geschichte, vol. 2, 2e éd., Fribourg/Tübingen, Laupp. Seemüller, J. (1904) : Die Doubletten in der ersten Dekade des Livius, Neuburg, Griessmayer. Seemüller, J. (1908) : Die Dubletten in der dritten Dekade des Livius, Neuburg, Griessmayer. Skutsch, O. (1985): The Annals of Q. Ennius, Oxford, Oxford University Press.

Syme, R.(2003) : Die Römische Revolution, rééd. Stuttgart, Klett-Cotta.

Tatum, W. J. (1999) : The Patrician Tribune. Publius Clodius Pulcher, Chapel Hill et al., UNC Press Books.

Thomas, Y. (1996) : « Rom : Väter als Bürger in einer Stadt der Väter », in : Burgière, A. et al. (éd.) : Geschichte der Familie I : Altertum, Francfort-sur-le-Main, Campus, p. 277-326.

Versnel, H. S. (1982) : « Die neue Inschrift von Satricum in historischer Sicht », Gymnasium, 89, p. 193-235.

Walter, U. (2001) : « Rollentausch und Übersetzung ins Absurde : Zur rhetorischen Strategie in der Rede des App. Claudius Crassus (Liv. 6, 40, 3-41) », Hermes, 129, p. 251-258.

Walter, U. (2002) : « Geschichte als Lebensmacht im republikanischen Rom », Geschichte in Wissenschaft und Unterricht, 53, p. 326-339.

Walter, U. (2004) : Memoria und res publica. Zur Geschichtskultur in der römischen Republik, Francfort-sur-le-Main, Verlag Antike.

Welwei, K.-W. (1993) : « Gefolgschaftsverband oder Gentilaufgebot? Zum Problem eines frühen familiare bellum (Liv. II 48, 9) », ZRG, 110, p. 60-76.

Welwei, K.-W. (2001) : « Lucius Iunius Brutus : Zur Ausgestaltung und politischen Wirkung einer Legende », Gymnasium 108, p. 123-135.

Wiseman, T. P.( 2003) : « The Legend of Lucius Brutus », in : Citroni, M. (dir.) : Memoria e identità. La cultura romana costruice la sua immagine, Florence, SAMERL, p. 21-38.

Wlosok, A. (1980) : « Nihil nisi ruborem. Über die Rolle der Scham in der römischen Rechtskultur », Grazer Beiträge, 9, p. 155-172.

Yacobson, A. (1999) : Elections and Electioneering in Rome, Stuttgart, Franz Steiner. 


\section{NOTES}

1. À cela correspondait un style de campagne électorale principalement centrée sur la personnalité, qui évitait en général les propositions programmatiques pour miser à la place sur la présence continue et la communication avec le plus grand nombre possible de personnes et de groupes importants. Voir le travail fondamental de M. Jehne (1995), p.60-63, qui présente d'autres preuves. Les campagnes électorales de Marius en 108 et de Pompée en 71 constituent des exceptions connues dans la république tardive ; contre l'idée selon laquelle les élections étaient en général dépolitisées quant à leur contenu, voir en particulier Yacobson (1999), chap. 6.

2. Voir à ce sujet l'article à la fois concis et brillant de G. Clemente (1990), en part. p. 240-245; en détail : Scholz (2011).

3. Sur l'importance constitutive du père comme exemple pratique et modèle socio-éthique, voir Thomas (1996), p. 277-326. Sur le lien entre les rôles dominants du père à la maison et dans la res publica, voir l'esquisse de J. Martin (2002a); voir aussi Martin (2002b).

4. Cf. Liv. praef. 10 ; à ce sujet, voir la contribution générale de J. Chaplin (2000).

5. Liv. 5, 18, 1-5 ; cf. MRR 1, p. 84-85 et 87-88. Cf. les pages essentielles de F. Münzer, RE 13.1 (1926), p. 234-236 s.v. Licinius (43); Ogilvie (1970), p. 666-669 avec d'autres indications bibliographiques. L'affirmation d'Ogilvie (p. 668), qui prétend que le discours qui suit le vote et précède la renonciation est « hors propos ", n'est bien entendu pas correcte. Le récit que l'on trouve chez Val. Max. 4, 5, 3 concernant les événements de l'an 175 est formellement identique ; cf. Mommsen (1887), p. 410 et note 3.

6. «En vobis » inquit « iuvenem », filium tenens, " effigiem atque imaginem eius, quem vos antea tribunum militum ex plebe primum fecistis. hunc ego institutum disciplina mea vicarium pro me rei publicae do dicoque vosque quaeso, Quirites, delatum mihi ultro honorem huic petenti meisque pro eo adiectis precibus mandetis. » = Liv. 5, 18, 5 [trad. Baillet mod.) n..d.t.].

7. On trouve des propos similaires concernant Scipion dans Liv. 26, 41, 24 : "Brevi faciam ut, quemadmodum nunc noscitatis in me patris patruique similitudinem oris voltusque et lineamenta corporis, ita ingenii fidei virtutisque effigiem vobis reddam ut revixisse aut renatum sibi quisque Scipionem imperatorem dicat. » Voir, avec une connotation négative, Cic. Sest. 17 : Quorum, per deos immortalis, si nondum scelera vulneraque inusta rei publicae vultis recordari, vultum atque incessum animis intuemini ; facilius eorum facta occurrent mentibus vestris, si ora ipsa oculis proposueritis. Voir aussi Cic. Mur. 16. M. Antoine était flatté de la ressemblance physique avec son ancêtre supposé, Hercule, tel que les gens le connaissaient par les statues et les portraits; voir Plut. Anton. 5, 1-4. Comme on le sait, Pompée n'était pas mécontent qu'on lui attribue une certaine ressemblance avec Alexandre, même si celle-ci était plus prétendue que réelle. (Plut. Pomp. 2, 1). [Trad. de la rédaction

8. Cicéron n'était pas le seul à être convaincu que maximas res publicas ab adulescentibus labefactatas, a senibus sustentatas et restitutas (esse) (Cato m. 20) [les plus grands États ont été renversés par des jeunes gens, soutenus ou redressés par des vieillards (trad. Wuilleumier). ; Pour une retractatio scientifique de cette idée v. J. Plescia (1976). La grande importance de la socialisation réussie des futurs dirigeants ressort également de l'article.

9. On trouve des renvois à la documentation chez Schwegler (1884), p. 45, note 2. - Travaux récents sur le personnage de Brutus en général : Welwei (2001), Wiseman (2003), tous deux avec des références plus anciennes.

10. Parmi les cas typiques dans lesquels le devoir civique «l'emporte sur les sentiments naturels du père » (Ihne [1893], p. 339, note 1), on peut citer, outre l'ensemble de légendes liées à Manlius Torquatus (voir plus loin), A. Postumius Tubertus, qui fit exécuter son fils pour non-respect de la discipline militaire ; voir Liv. 4, 26, 1-29, 8 ; Ogilvie (1970), p. 576-577. - La haute considération dont un tel acte jouissait auprès des Romains représentait aux yeux d'un observateur attentif un trait culturel unique et marquant ; voir Dion. Hal. ant. 5, 8, 1. 
11. Même un homo novus, dont les ancêtres n'étaient par définition pas "visibles ", pouvait se réclamer de cette sémantisation et de cet idéal de ressemblance; voir Cic. leg. agr. 2, 1 : Mihi, Quirites, apud vos de meis maioribus dicendi facultas non datur, non quo non tales fuerint quales nos illorum sanguine creatos disciplinisque institutos videtis, sed quod laude populari atque honoris vestri luce caruerunt. [Quant à moi, Quirites, je ne peux parler devant vous de mes ancêtres, non pas qu'ils furent différents de ce que vous me voyez être, moi qui suis né de leur sang et ai été éduqué par leurs leçons, mais parce qu'ils n'ont pas bénéficié de la gloire auprès du peuple et de l'éclat qu'apportent vos honneurs; trad. dans M. Jacotot: Question d'honneur. Les notions d'honos, honestum et honestas dans la République romaine antique, Rome, Publications de l'École française de Rome, 2013, en ligne].

12. Cf. Bradley (1991), p. 138-139: «From the moment of birth the life of the upper-class Roman child was potentially subject to a high degree of emotional uncertainty and dislocation, the product of such factors as early separation from a natural parent by death or divorce, periodic separation from a father because of the demands of a public career or political crisis, the abrupt organization of new households, and conjunction with stepparents and stepsiblings occasioned by parental remarriage. "

13. Voir, pour une étude de cas, Walter (2001).

14. Voir à ce sujet Flaig (1995); (2003), p. 49-68.

15. Cf. Schwegler (1884), p. 57-59; Linke (1995), p. 154-155 avec sources et bibliographie.

16. Cf. Syme (2003), p. 26 ; Tatum (1999), p. 32 et passim ; Walter (2004), p. 121-130. Mommsen (1864) reste important.

17. Aussi la plèbe, au vu de l'attitude hostile de Caeso Quintus (Céson) à son égard, est-elle consternée par le choix de son père, L. Quinctius Cincinnatus, comme consul suffect : iratum, potentem favore patrum, virtute sua, tribus liberis, quorum nemo Caesoni cedebat magnitudine (Liv. 3, $19,3)$.

18. Voir Schwegler (1884), p. 494-498, 502-504; on trouve un tableau généalogique chez Beloch (1926), p. 54. A. Drummond, $\mathrm{CAH}^{2} 7.2$ (1989) p. 184 et 207 signale qu'immédiatement après la «chute» de Sp. Cassius en 486, plusieurs familles éminentes sont apparues sur la scène politique : outre les Fabii, il y a eu les Cornelii, les Aemilii, les Manlii et les Quinctii. - Fabium nomen: Liv. $2,42,2.8 ; 2,45,16$. Voir en particulier, concernant la solidarité entre frères particulièrement encouragée par le mos maiorum - un phénomène qui complète celui que nous expliquons ici - voir Bannon (1997), en particulier p. 138-148.

19. À ce sujet, voir en particulier Pais (1906), p. 168-184; Richard (1989), (1990). Sur l'éventuelle strate réellement historique, voir Welwei (1993). - Il faut noter que les formes de liens de coopération ou de clientèle] des débuts de l'histoire italo-romaine telles que le Lapis Satricanus les atteste dans les « compagnons de Mars » de Poplios Valesios (voir à ce sujet Versnel [1982] avec bibliographie antérieure) n'ont quasiment pas laissé de traces dans les documents anciens; le paradigme gentilice-familial domine presque entièrement.

20. Voir Liv. 5, 46, 1-3 ; 52, 3 ; Val. Max. 1, 1, 11 ; Cassius Hemina, FRH 6 F 22 avec commentaire.

21. Voir à ce propos la contribution détaillée de Beck (2005), p. 269-301.

22. Val. Max. 5, 7, 1.

23. Voir 10, 3, 7 : Qui terror non eo tantum a Fabio abhorret, quod, si qua alia arte, cognomen suum aequavit [tum] maxume bellicis laudibus, sed etiam quod memor Papirianae saevitiae numquam, ut dictatoris iniussu dimicaret, adduci potuisset. - Les fastes capitolins nomment d'abord Aemilius, puis Fabius ; cf. MRR 1, 170.

24. Voir Liv. 4, 2, 7 (Icilios = « Des gens comme Icilius »); 52, 2 (L. Icilius ... cum principio statim anni velut pensum nominis familiaeque seditiones agrariis legibus promulgandis cieret); 54, 4 (familia infestissima patribus). Cette certitude historique relative a été reconstituée par F. Münzer, RE 9.1 (1916), p. 850-855, s.v. Icilius (où on trouve aussi tous les renvois à la documentation); on peut certainement juger justifiée son hypothèse suivant laquelle la tradition contaminée à plusieurs 
reprises qui répartit les récits distincts de manière assez arbitraire entre les différents porteurs du nom doit avoir un fondement historique dans la mesure où les Icilii ont complètement disparu de la politique après 409 , ce qui rend improbable l'hypothèse d'une pure invention à une époque ultérieure (par qui aurait-elle pu voir le jour ?). Concernant la lex Icilia de Aventino publicando (qui date apparemment de 456), voir Flach (1994), p. 95-98. - L'importance du nom, y compris au sein de la plèbe, entraîne aussi le choix de $\mathrm{C}$. Sicinius comme tribun de la plèbe après le second décemvirat en tant que progeniem eius quem primum tribunum plebis creatum in Sacro monte proditum memoriae est (Liv. 3, 54, 12).

25. L'intervention collective d'hommes du même genre pouvait apparemment être considérée comme un trait caractéristique de la politique aristocratique; cf. Liv. 4, 55, 3 : Tres erant (scil. Icilii) et omnes acerrimi viri generosique iam, ut inter plebeios.

26. Beck (2005), p. 229-243.

27. Liv. 5, 11, 1: qui nomini ac familiae debitum praestare videretur Treboniae legis patrocinium. Au sujet du plebiscitum Trebonium (semble-t-il de 448), voir Liv. 3, 65, 1-4; Flach (1994), p. 227-228 avec renvois aux documents et bibliographie - Un autre tribun de la plèbe, Sp. Maelius, tenta d'accuser les deux patriciens qui avaient semble-t-il poursuivi et assassiné son père, favore nominis moturum se aliquid ratus, ne fut toutefois pas pris au sérieux ; cf. Liv. 4, 21, 3-4.

28. Cette reconstitution résulte de l'harmonisation de Liv. 8, 28, 8-9 et de Varr. ling. 7, 105. À propos de Poetelius Libo pater, voir MRR 1, p. 120, 131 et 146-147. Le contenu exact de la lex Poetelia Papiria de nexis de 326 est sujet à controverse ; cf. Hölkeskamp (1987), p. 159-160 ; Cornell (1995), p. 330-333 ; Oakley (1998), p. 688-691 ; Elster (2003), p. 63-71, tous avec une bibliographie mise à jour - Varron attribue à Poetelius Libo, le fils de celui que nous venons de nommer, l'abrogation complète du nexum en lien avec sa dictature de 313 (qui n'était d'ailleurs probablement qu'une clavi figendi causa) ; Tite-Live ne le signale pas. Beloch (1926), p. 70 juge vraisemblable, étant donné le grande espacement des consulats du père, que celui de 326 appartienne au fils, et qu'il n'y ait donc qu'une seule loi, proposée par le fils; voir aussi Oakley (1998), p. 149-150. Un intervalle si long n'était toutefois pas sans précédent; T. Manlius Torquatus (voir plus loin) fut consul en 347, 344, 340 ainsi que dictateur en 320. Quoiqu'il en soit, cette question de la réalité des faits est d'importance secondaire pour le modèle d'engendrement d'une tradition.

29. Renvois à la documentation et références chez Flach (1994), p. 59-62; titres importants : Bleicken (1959) ; Lintott (1972); Jehne (2002).

30. Cf. Flach (1994), p. 216-218.

31. Cf. le travail détaillé d'Elster (2003), p. 98-103.

32. Clairement formulé par P. Valerius Publicola (cos. I 475) : memorem cognominis quo populi colendi velut hereditaria cura sibi a maioribus tradita esset, concilium plebis non impediturum (Liv. 3, 18, 6).

33. Au sujet de la mémoire centrée sur la famille, voir à présent Blösel (2003) et Walter (2004), chap. 3. Les conditions de fonctionnement d'une telle tradition familiale sont citées chez Cic. Flacc. 25 : Sed cum L. Flacci res agatur ex <ea> familia, cuius qui primus consul est factus primus in hac civitate consul fuit (le père fondateur marquant, inoubliable), cuius virtute regibus exterminatis libertas in re publica constituta est (son acte constituant) quae usque ad hoc tempus honoribus imperiis rerum gestarum gloria continuata permansit (conservation permanente du profil donné à ses actions), cumque ab hac perenni contestataque virtute maiorum non modo non degeneraverit $L$. Flaccus, sed id quod maxime florere in generis sui gloria viderat laudem patriae in libertatem vindicandae (le catchword familial) praetor adamarit (existence dans le présent également d'un descendant adéquat).

34. Cf. les collectes de J. Seemüller (1904) et (1908). La discussion de Oakley (1997), p. 102-104, est importante, y compris pour ce qui suit. 
35. Cela se vérifie encore pour des époques historiquement mieux connues; au sujet du double combat de cavalerie dans la guerre d'Hannibal (Liv. 29, 28, 10-29, 4 ; 29, 34-35), l'auteur observe lui-même $(29,35,2)$ : duos eodem nomine Carthaginiensium duces duobus equestribus proeliis interfectos non omnes auctores sunt, veriti, credo, ne falleret bis relata eadem res. Dans l'examen plus ancien des sources, qui procédait de manière mécanique, ce genre d'erreur était généralement expliqué par un changement de modèle potentiel.

36. Cf. Beloch (1926), p. 401-402 avec les renvois à la documentation. C. Iunius Brutus Bubulcus, consul en 291 et en 277, triumpha des Lucanii et des Bruttii (InscrIt 13, 1, 73).

37. Cela apparaît de façon frappante chez Oakley (1997), p. 103. Il en va de même pour M. Antonius père et fils, qui ont exercé en 102-100 et en 74 respectivement des commandements extraordinaires contre les pirates en Méditerranée orientale.

38. Cf. Cic. rep. 2, 54 : neque vero leges Porciae, quae tres sunt trium Porciorum, ut scitis, quicquam praeter sanctionem attulerunt novi. De ces lois, qui traitaient des châtiments corporels à l'encontre des citoyens romains (Liv. 10, 9, 4 ; 300 av. J.-C.) et dont le rapport avec les lois de provocation valérienne n'est pas clair, seules deux sont documentées indépendamment, toutes deux presque en même temps au début du II ${ }^{\mathrm{e}}$ siècle ; cf. Festus p. 266 Lindsay (conplures leges) ; J. Bleicken, RE 23.1 (1957), col.] 2444-2464 s.v. provocatio, ici : col. 2448 ; Jehne (2002), p. 64-65 ; Elster (2003), p. 296-301.

39. Occurrences principales : Liv. 8, 6, 9-13 et 8, 9, 4-14 (340) ; 10, 28, 6-29, 4 (295) ; Cic. fin. 2, 61 ; Tusc. 1, 89 ; cf. MRR 1, p. 192-193 (279) ; pour des preuves plus récentes et une analyse détaillée, voir F. Münzer, RE 4.2 (1901), col. 2279-2286 s.v. Decius (15-17) ; voir aussi, en priorité, Oakley (1997), p. 477-505.

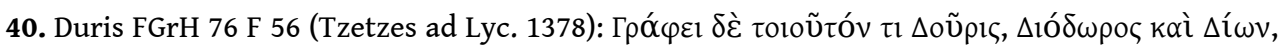

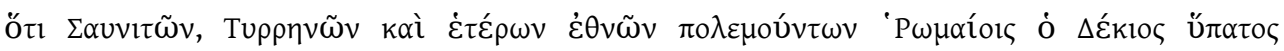

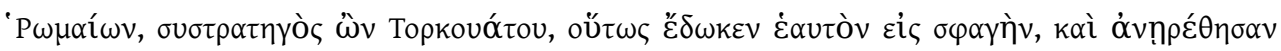

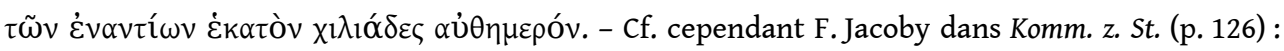
«La citation n'offre aucune certitude quant au fait de savoir si D(uris) a rapporté le sacrifice mortel du consul. »

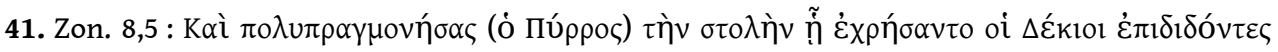

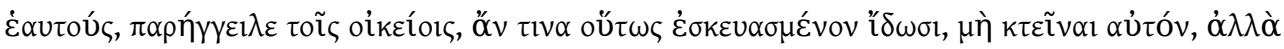

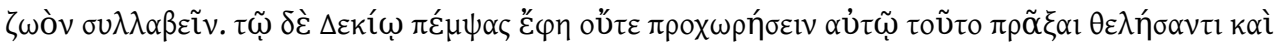

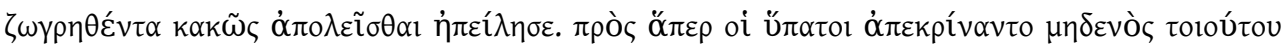

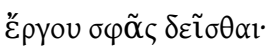

42. Accius F 15 Ribbeck $^{3}$ : Patrio exemplo et me dicabo atque animam devoro hostibus; voir aussi Liv. 10, 7, 3-4. [Trad. fr. : Charles Guittard, « Tite-Live, Accius et le rituel de la devotio », Comptes rendus des séances de l'Académie des Inscriptions et Belles Lettres, 1984, p. 593.] - Ennius : Ann. 191-194 Skutsch. Le numéro du volume transmis (VI) montre qu'Ennius parle ici du troisième Decius; Cicéron pourrait l'avoir pris comme source d'information lorsqu'il parle de trois dévotions (fin. 2, 61 ; Tusc. 1, 89; ce lien avec Ennius était été présumé par Niebuhr [1843], p. 592). Cf. à propos d'Ennius les observations importantes de Skutsch (1985), p. 353-355. - Les Decii étaient si connus que Cicéron pouvait même les utiliser dans une inversion parodique; cf. Phil. 11, 13 : Vidi etiam P. Deci auctionem, clari viri, qui maiorum exempla persequens pro alieno se aere devovit.

43. MRR $1,202^{2}$; cf. Oakley (1998), p. 478-479 avec une discussion intéressante. Val. Max. 5, 6, 5-6 ne cite que les deux premières dévotions.

44. Il a pourtant parfois été attribué à la force de la prophétisation de la mort et du malheur ; cf. A. Steier, RE, 14.2 (1930), col. 2396-2408 (2406-2407).

45. Liv. 10, 7, 2-4: Rettulisse dicitur Decius parentis sui speciem, qualem eum multi, qui in contione erant, viderant, incinctum Gabino cultu super telum stantem, quo se habitu pro populo ac legionibus Romanis devovisset: tum P. Decium consulem purum piumque deis inmortalibus visum, aeque ac si T. 
Manlius collega eius devoveretur; eundem P. Decium, qui sacra publica populi Romani faceret, legi rite non potuisse?

46. Cf. Cic. off. 3, 112 ; Liv. 7, 5 ; Val. Max. 5, 4, 3 ; App. Samn. 2 ; Sen. benef. 3, 37, 4 ; Vir. ill. 28, 1-2.

47. Plus ancienne description : Claudius Quadrigarius FRH $14 \mathrm{~F} 10 \mathrm{~b}$.

48. Mention principale : Liv. 7, 1-22 ; cf. Oakley (1998), p. 436-451; d'autres preuves sont exposées dans le même ouvrage, p. 436. - L'exécution de son propre fils par A. Postumius Tubertus (Liv. 4, $29,5-6)$ est en fait analogue.

49. Cf. Oakley (1998), p. 427 au sujet de l'association des exempla par Tite-Live. C'est clairement le consul qui punit, et non le père.

50. Cic. fin. 1, 24 : Pronuntiaret eum non talem videri fuisse in imperio, quales eius maiores fuissent, et in conspectum suum venire vetuit; voir Liv. epit. 54 ; Val. Max. 5, 8, 3 ; F. Münzer, RE 14.1 (1928), col. 1209-1210 s.v. Manlius (83) et les autres attestations; à propos de l'interprétation, Wlosok (1980), p. 165-172 ; Flaig (2003), p. 78-82.

51. Cf. Bettini (1991), p. 6-9 (également pour les lignes qui suivent).

52. Expressément confirmé pour l'exécution de 340 ; cf. Front. strat. 4, 1, 41: Manlius filius, exercitu pro se adversus patrem seditionem parante, negavit tanti esse quemquam, ut propter illum disciplina corrumperetur, et obtinuit, ut ipsum puniri paterentur.

53. Il mentionne en 22, 60, 11 les sauvetages du premier P. Decius Mus en tant que tribun militaire durant la guerre samnite et du tribun - anonyme chez Caton - M. Calpurnius Flamma (plus exactement Q. Caedicius; cf. le commentaire de Caton FRH 3 F 4,7a), qui sauva en 258 l'armée romaine d'un encerclement mortel près de Camarine. - Le rachat controversé de prisonniers était traité dans l'historiographie romaine dès le II ${ }^{\mathrm{e}}$ siècle av. J.-C. ; voir Acilius FRH 5 F 5 .

54. Oakley (1998), p. 428 n'exclut pas l'historicité du duel et du châtiment consécutif du fils.

55. Cicéron a ainsi tenté d'amener le futur triumvir Lépide à une politique loyale envers le sénat en faisant référence à son arrière-grand-père, qui portait le même nom : Phil. 13, 14.

56. Au sujet de la succession de Censorius, voir Fehrle (1983), p. 50, p. 68-69, p. 70 note 27, p. 90 avec note 29, p. 111 note 133, p. 178 , avec à chaque fois des indications bilbliographiques supplémentaires.

57. Au sujet de Brutus, voir Walter (2002), p. 334-339 avec d'autres références.

58. Bien entendu, les actes et le profilage au sein de la noblesse n'étaient pas toujours dominés par la formation d'une identité familiale aussi forte que chez les Claudii patriciens ; ainsi les Acilii Glabriones, des plébéiens, faisaient-ils passer l'identité de groupe avant celle de la famille et ne tentaient pas d'imposer un « programme " politique propre; cf. Dondin-Payre (1993).

59. [J'ai, de manière très embarrassante, raté en son temps l'essai de J.-C. Richard [1972] ; on trouve une traduction en anglais avec addendum in : Richardson / Santangelo (2014), p. 239-257. Richard parle de Ti. Sempronius Gracchus (tr. pl. 133) et de son père (cos. I 177).]

\section{INDEX}

Mots-clés : République romaine, nobilitas, socialisation

Schlüsselwörter : römische Republik, nobilitas, Sozialisierung 


\section{AUTEURS}

\section{UWE WALTER}

Uwe Walter est professeur d'histoire ancienne à l'Université de Bielefeld. Pour plus d'informations, voir la notice suivante. 